\title{
Modified Inverse Rayleigh Distribution
}

\author{
Muhammad Shuaib Khan \\ School of Mathematical and \\ Physical Sciences \\ The University of Newcastle \\ Callaghan, NSW 2308, \\ Australia
}

\begin{abstract}
A two parameter generalization of the Inverse Rayleigh distribution capable of modeling bathtub hazard rate function is defined and studied with application to reliability data. A comprehensive account of the mathematical properties of the modified Inverse Rayleigh distribution including estimation and simulation with its reliability behavior are discussed. An application is presented to illustrate the proposed distribution.
\end{abstract}

\section{Keywords}

Reliability functions; moment estimation; moment generating function; order statistics; maximum likelihood estimation

\section{INTRODUCTION}

The modified inverse Rayleigh (MIR) distribution is the special case of the modified inverse Weibull (MIW) distribution proposed by Khan M. S and King R [7] and studied its theoretical properties. The modified inverse Rayleigh distribution approaches to the inverse Rayleigh and inverse exponential distributions when its parameters changes. The modified inverse Rayleigh distribution is very useful lifetime model which can be used for analyzing lifetime data. In this research the properties of the modified inverse Rayleigh distribution are discussed. Trayer [12] introduced the Inverse Rayleigh Distribution. Gharraph. [5], Mukarjee and Maitim [11] studied some properties of the inverse Rayleigh distribution. Voda [13, 14, 15] also discussed some properties of the maximum likelihood estimator for the Parameter $\theta$ of the inverse Rayleigh distribution. A Comparison of the negative moment estimator with maximum likelihood estimator of the inverse Rayleigh distribution studied by Mohsin and Shahbaz [10]. The cumulative distribution function (cdf) of the inverse Rayleigh distribution is given by

$$
G(x ; \theta)=\exp \left\{-\theta\left(\frac{1}{x}\right)^{2}\right\}
$$

The probability density function (pdf) corresponding to (1) is

$$
g(x ; \theta)=\frac{2 \theta}{x^{3}} \exp \left\{-\theta\left(\frac{1}{x}\right)^{2}\right\}
$$

Here $\theta$ is the scale parameter. The behavior of instantaneous failure rate of the inverse Rayleigh distribution has increasing and decreasing failure rate patterns for lifetime data.

\section{MODIFIED INVERSE RAYLEIGH DISTRIBUTION}

Let $x$ be a random variable having the MIR distribution with parameters $\alpha$ and $\theta$ then the probability density function given in (3) with their associated cumulative distribution function, reliability function, hazard function and cumulative hazard function are given in (4-7) respectively

$$
\left.\begin{array}{c}
f(x)=\left(\alpha+\frac{2 \theta}{x}\right)\left(\frac{1}{x}\right)^{2} \exp \left\{-\frac{\alpha}{x}-\theta\left(\frac{1}{x}\right)^{2}\right\} \\
F(x)=\exp \left\{-\frac{\alpha}{x}-\theta\left(\frac{1}{x}\right)^{2}\right\} \\
R(x)=1-\exp \left\{-\frac{\alpha}{x}-\theta\left(\frac{1}{x}\right)^{2}\right\} \\
h(x)=\frac{\left(\alpha+\frac{2 \theta}{x}\right)\left(\frac{1}{x}\right)^{2} \exp \left\{-\frac{\alpha}{x}-\theta\left(\frac{1}{x}\right)^{2}\right\}}{1-\exp \left\{-\frac{\alpha}{x}-\theta\left(\frac{1}{x}\right)^{2}\right\}} \\
H(x)=-\ln \mid \exp \left\{-\frac{\alpha}{x}-\theta\left(\frac{1}{x}\right)^{2}\right\} \\
H(x)=\int_{0}\left(\frac{2 \theta}{x}\right)\left(\frac{1}{x}\right)^{2} \exp \left\{-\frac{\alpha}{x}-\theta\left(\frac{1}{x}\right)^{2}\right\} \\
(x)
\end{array}\right\}
$$

Here $\alpha$ and $\theta$ are the scale parameters representing the different patterns of the MIR distribution. Fig. 1a shows the different patterns of the MIR distribution. The non-reliability and reliability patterns are shown in Fig. $1 b$ and Fig. 2a The behavior of instantaneous failure rate of the MIR distribution has upside-down bathtub shape curve in Fig. $2 b$. 


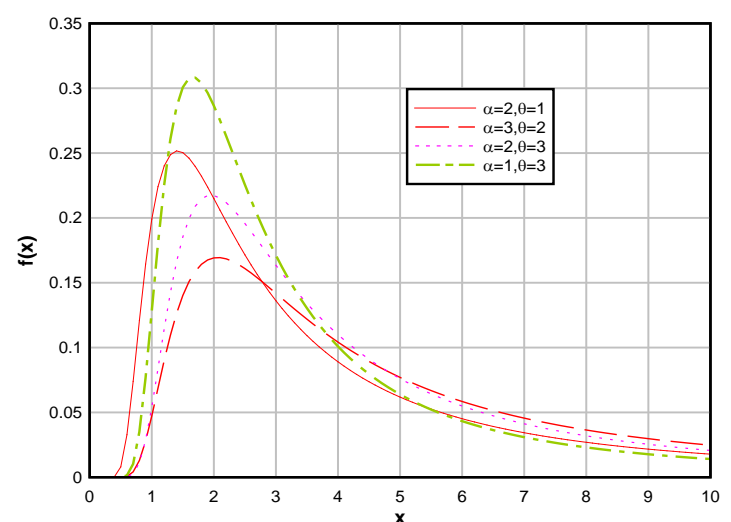

(a) Density Function

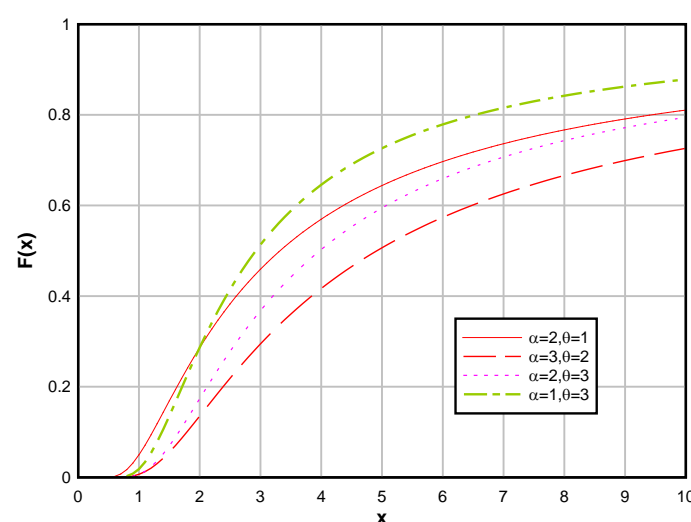

(b) Distribution Function

Fig 1:The Modified Inverse Rayleigh PDF \& CDF

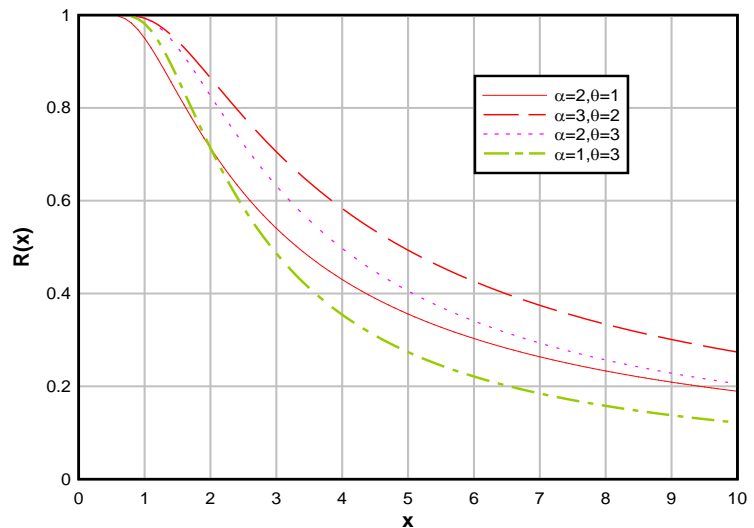

(a) Reliability Function

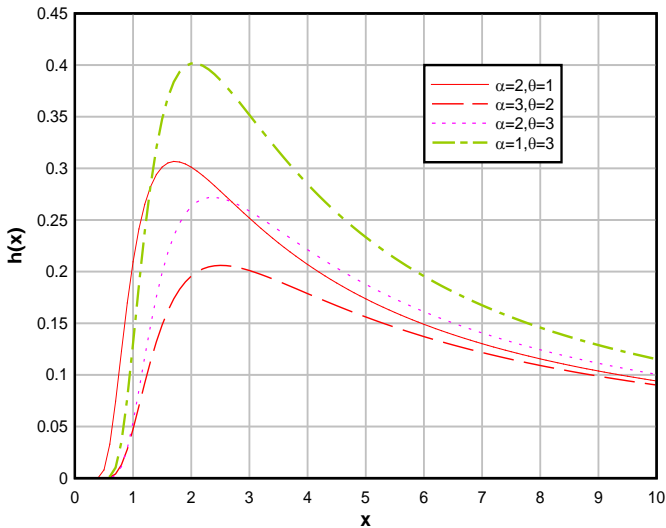

(b) Hazard Function

Fig 2:The Modified Inverse Rayleigh RF \& HF

\section{STATISTICAL PROPERTIES}

This section explain the statistical properties of the MIR distribution.

\subsection{Quatntile and Median}

The quantile $x_{q}$ of the MIR distribution is the real solution of the following equation.

$$
x_{q}=\frac{2 \theta}{-\alpha+\sqrt{\alpha^{2}-4 \theta \ln (q)}}
$$

The above equation has the closed form solution in $x_{q}$. By substituting $\mathrm{q}=0.5$, one can obtain the median of the MIR distribution. Fig. $3 a$ and $3 b$ shows the median and quartile deviation life of the MIR distribution. To illustrate the skewness and kurtosis we consider the measure based on quantiles. The skewness and kurtosis measures can now be calculated from quantiles using Bowley $(\mathcal{B})[6]$ and Percentile coefficient of kurtosis well known relationships. The Bowley Skewness and Percentile coeffficient of kurtosis when $\alpha=2$, as a function of $\theta$ are illustrated in Fig. $4 a$ and $4 b$. The Bowley's skewness is given by

$$
\mathcal{B}=\frac{\mathcal{Q}(3 / 4)+\mathcal{Q}(1 / 4)-2 \mathcal{Q}(2 / 4)}{\mathcal{Q}(3 / 4)-\mathcal{Q}(1 / 4)}
$$

The Percentile coefficient of kurtosis is given by

$$
\mathcal{P}=\frac{\mathcal{Q}(3 / 4)-\mathcal{Q}(1 / 4)}{2[\mathcal{Q}(9 / 10)-\mathcal{Q}(1 / 10)]}
$$

The behavior of the Bowley $(\mathcal{B})$ skewness is decreasing as the parameter $\theta$ increases is illustrated in Fig. 4 a. Fig. $4 \mathrm{~b}$ shows the pattern of the Percentile coefficient of kurtosis is increasing as the parameter $\theta$ increases.

\subsection{Random Number Generation}

The random number as $\mathrm{x}$ of the $\operatorname{MIR}(\mathrm{x} ; \alpha, \theta)$ is defined by the following equation

$$
x=\frac{2 \theta}{-\alpha+\sqrt{\alpha^{2}-4 \theta \ln (\omega)}}
$$

where $\omega \sim \mathrm{U}(0,1)$

The above equation is in closed form solution in $\mathrm{x}$, using $\omega$, a random number is uniformly distributed from zero to one, we have solved the above equation $\mathrm{F}(\mathrm{x})=\omega$ to obtain a random number in $\mathrm{x}$. 


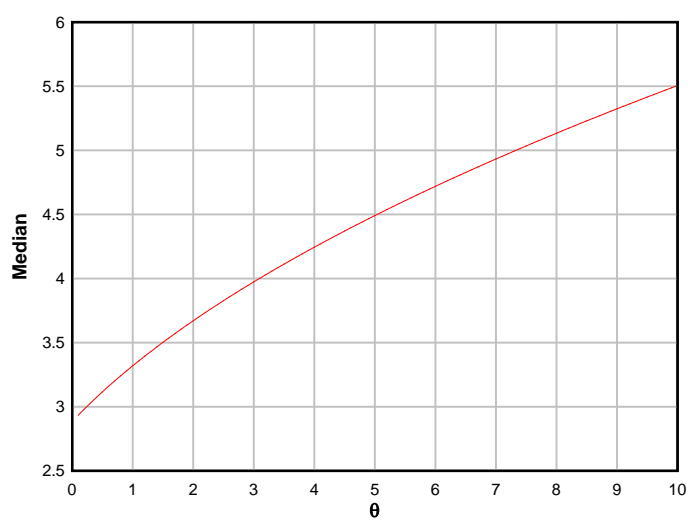

(a) Median

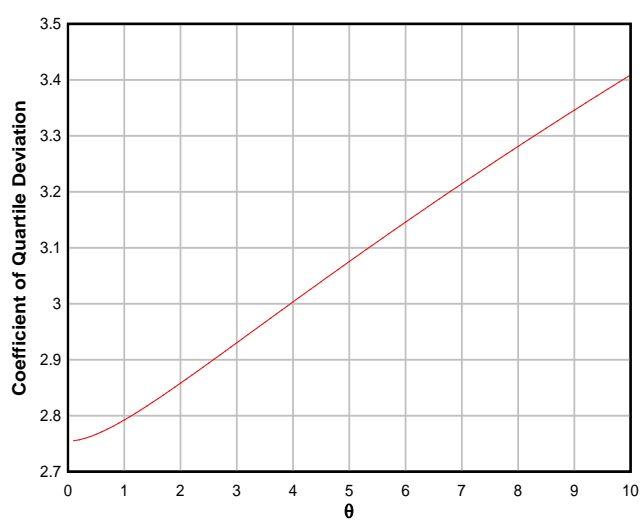

(b) Coefficient of Quartile Deviation

Fig 3:The Modified Inverse Rayleigh Quantiles

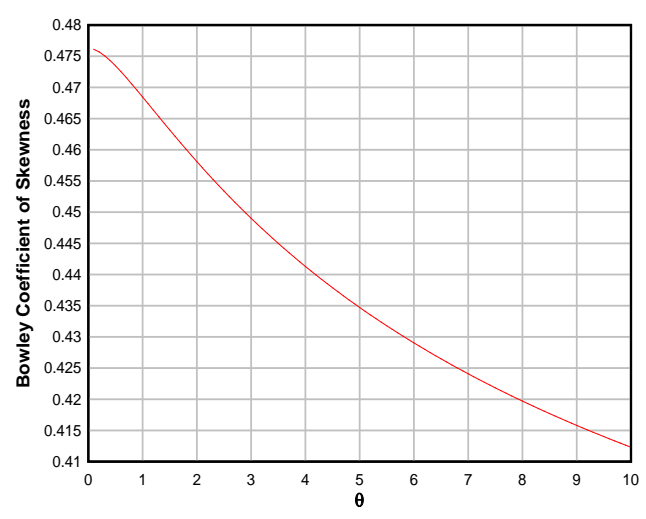

(a) Bowley,s Skewness

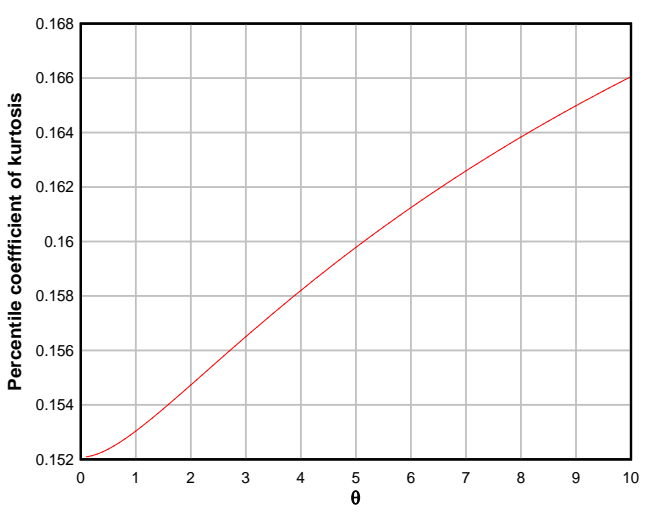

(b) Percentile coefficient of kurtosis

Fig 4: The Modified Inverse Rayleigh Skewness and Kurtosis

\subsection{Moments}

This subsection present the $\mathrm{k}^{\text {th }}$ moment, moment generating function of the $\operatorname{MIR}(\mathrm{x} ; \alpha, \theta)$ distribution.

Theorem 1: If $\mathrm{X}$ has the $\operatorname{MIR}(\mathrm{x} ; \alpha, \theta)$, then the $\mathrm{k}^{\text {th }}$ moment of $\mathrm{X}, \mu_{\mathrm{k}}$ is given as follows

$$
\mu_{k}=\int_{0}^{\infty} x^{k-2}\left(\alpha+\frac{2 \theta}{x}\right) \exp \left\{-\frac{\alpha}{x}-\theta\left(\frac{1}{x}\right)^{2}\right\} d x
$$

Proof:

$$
\begin{aligned}
& \mu_{k}=\alpha \int_{0}^{\infty} x^{k-2} \exp \left\{-\frac{\alpha}{x}-\theta\left(\frac{1}{x}\right)^{2}\right\} d x \\
& \quad+2 \theta \int_{0}^{\infty} x^{k-3} \exp \left\{-\frac{\alpha}{x}-\theta\left(\frac{1}{x}\right)^{2}\right\} d x \\
& \mu_{k}=\sum_{m=0}^{\infty} \frac{(-1)^{m} \theta^{m}}{m !} \alpha^{k-2 m} \mathfrak{\Im}(\alpha, \theta, k, m)
\end{aligned}
$$

$$
\mathfrak{I}(\alpha, \theta, k, m)=\left[\Gamma(2 m-k+1)+\frac{2 \theta}{\alpha^{2}} \Gamma(2 m-k+2)\right]
$$

Theorem 2: If $\mathrm{X}$ has the $\operatorname{MIR}(\mathrm{x} ; \alpha, \theta)$, then the moment generating function (mgf) of $X, M_{X}(t)$ is given as follows

$M_{x}(t)=\int_{0}^{\infty}\left(\alpha+\frac{2 \theta}{x}\right)\left(\frac{1}{x}\right)^{2} \exp \left\{t x-\frac{\alpha}{x}-\theta\left(\frac{1}{x}\right)^{2}\right\} d x$

Proof:

$$
\begin{aligned}
& M_{x}(t)=\alpha \int_{0}^{\infty}\left(\frac{1}{x}\right)^{2} \exp \left\{t x-\frac{\alpha}{x}-\theta\left(\frac{1}{x}\right)^{2}\right\} d x \\
& +2 \theta \int_{0}^{\infty}\left(\frac{1}{x}\right)^{3} \exp \left\{t x-\frac{\alpha}{x}-\theta\left(\frac{1}{x}\right)^{2}\right\} d x \\
& M_{x}(t)=\sum_{m=0}^{\infty} \sum_{n=0}^{\infty} \frac{(-1)^{m} \theta^{m} t^{n}}{m ! n !} \alpha^{n-2 m} \mathfrak{R}(\alpha, \theta, m, n)(13)
\end{aligned}
$$

where 
$\mathfrak{R}(\alpha, \theta, m, n)=\left[\Gamma(2 m-n+1)+\frac{2 \theta}{\alpha^{2}} \Gamma(2 m-n+2)\right]$

\subsection{Entropy and Mean Deviation}

The entropy of a random variable $\mathrm{X}$ with probability density $f(x)$ is a measure of variation of the uncertainty. A large value of entropy indicates the greater uncertainty in the data. The Rényi entropy is defined as

$$
I_{R}(\rho)=\frac{1}{1-\rho} \log \left\{\int f(x)^{\rho} \mathrm{d} x\right\}
$$

For the convince, let $u(x)=\int f(x)^{\rho} \mathrm{d} x$, is given by

$$
\begin{aligned}
& u(x)=\int_{0}^{\infty}\left(\alpha+\frac{2 \theta}{x}\right)^{\rho}\left(\frac{1}{x}\right)^{2 \rho} \exp \left\{-\frac{\rho \alpha}{x}-\theta \rho\left(\frac{1}{x}\right)^{2}\right\} d x \\
& u(x)=\sum_{g=0 h=0}^{\infty} \sum^{\infty} \frac{(-1)^{h}(\theta \rho)^{h} \alpha^{\rho}}{h !}\left(\begin{array}{l}
\rho \\
g
\end{array}\right)\left(\frac{2 \theta}{\alpha}\right)^{g} v(\alpha, \rho, g, h) \\
& \text { where } v(\alpha, \rho, g, h)=\int_{0}^{\infty}\left(\frac{1}{x}\right)^{2 \rho+2 h+g} \exp \left\{-\frac{\rho \alpha}{x}\right\} d x \\
& u(x)=\sum_{g=0 h=0}^{\infty} \sum^{\infty} \frac{(-1)^{h}(\theta \rho)^{h} \alpha^{\rho}}{h !}\left(\begin{array}{l}
\rho \\
g
\end{array}\right)\left(\frac{2 \theta}{\alpha}\right)^{g} \Gamma(2(\rho+h)+g-1) \\
& I_{R}(\rho)=\frac{\rho}{1-\rho} \log (\alpha)+\frac{1}{1-\rho} \log \left\{\sum_{g=0 h=0}^{\infty} \sum^{\infty} \frac{(-1)^{h}(\theta \rho)^{h} \alpha^{\rho}}{h !}\right. \\
& \left.\left(\begin{array}{l}
\rho \\
g
\end{array}\right)\left(\frac{2 \theta}{\alpha}\right)^{g}\left(\frac{1}{\alpha \rho}\right)^{2(\rho+h)+g-1} \Gamma(2(\rho+h)+g-1)\right\}
\end{aligned}
$$

If $\mathrm{X}$ has the $\operatorname{MIR}(\mathrm{x} ; \alpha, \theta)$ distribution, then we can derive the mean deviation about mean and about the median $\mathrm{M}$ can be obtain from the following equations Gauss et al. [4]

$$
\delta_{1}=\mu \mathrm{F}(\mu)-\psi(\mu) \quad \text { and } \quad \delta_{2}=\mu-2 \psi(\mathrm{M})
$$

The mean is obtained from (12) with $k=1$ and the median M is the solution of the non-linear equation is obtained from (8), where $\psi(q)$ can be obtained from (3)

$$
\psi(q)=\sum_{s=0}^{\infty} \frac{(-1)^{s} \theta^{s}}{s !}\left(\frac{1}{\alpha}\right)^{2 s} w(\alpha, \theta, q, s)
$$

where

$$
w(\alpha, \theta, q, s)=\gamma\left(2 s+1, \frac{\alpha}{q}\right)+\frac{2 \theta}{\alpha^{2}} \gamma\left(2 s+2, \frac{\alpha}{q}\right)
$$

Using (17), the equation of Bonferroni [3] and Lorenz [9] curves are given in (18) and (19).

$$
\begin{aligned}
& B(q)=\frac{1}{P \mu} \sum_{s=0}^{\infty} \frac{(-1)^{s} \theta^{s}}{s !}\left(\frac{1}{\alpha}\right)^{2 s} w(\alpha, \theta, q, s)(18) \\
& L(q)=\frac{1}{P} \sum_{s=0}^{\infty} \frac{(-1)^{s} \theta^{s}}{s !}\left(\frac{1}{\alpha}\right)^{2 s} w(\alpha, \theta, q, s)
\end{aligned}
$$

where $q=\psi(p)$ is calculated from (9)

\section{ORDER STATISTICS}

Let $\mathrm{x}_{1}, \mathrm{x}_{2}, \ldots, \mathrm{x}_{\mathrm{n}}$ are independently identically distributed ordered random variables from the $\operatorname{MIR}(\mathrm{x} ; \alpha, \theta)$ distribution having Ist order and nth order probability density function is

$$
\begin{aligned}
& \mathrm{f}_{1: \mathrm{n}}(\mathrm{x})=\mathrm{C}_{\mathrm{r}: \mathrm{n}}(1-\mathrm{F}(\mathrm{x}))^{\mathrm{n}-1} \mathrm{f}(\mathrm{x}) \\
& \mathrm{f}_{\mathrm{n}: \mathrm{n}}(\mathrm{x})=\mathrm{C}_{\mathrm{r}: \mathrm{n}}(\mathrm{F}(\mathrm{x}))^{\mathrm{n}-1} \mathrm{f}(\mathrm{x})
\end{aligned}
$$

By substituting (3) and (4) in above equations is given by

$$
\begin{aligned}
f_{1: n}(x)=n\left(\alpha+\frac{2 \theta}{x}\right)\left(\frac{1}{x}\right)^{2} \exp \left\{-\frac{\alpha}{x}-\theta\left(\frac{1}{x}\right)^{2}\right\} \\
\times\left[1-\exp \left\{-\frac{\alpha}{x}-\theta\left(\frac{1}{x}\right)^{2}\right\}\right]^{n-1} \\
f_{n: n}(x)=n\left(\alpha+\frac{2 \theta}{x}\right)\left(\frac{1}{x}\right)^{2} \exp \left\{-\frac{\alpha}{x}-\theta\left(\frac{1}{x}\right)^{2}\right\}^{n-2}
\end{aligned}
$$

Theorem 3: If $X$ has the $\operatorname{MIR}(\mathrm{x} ; \alpha, \theta)$, then the pdf of the $\mathrm{k}^{\mathrm{th}}$ order statistics $\mathrm{X}_{(\mathrm{r})}$ is given by

$$
f_{r: n}(x)=\frac{(F(x))^{r-1}(1-F(x))^{n-r} f(x)}{B(r, n-r+1)}
$$

where $\mathrm{B}(.,$.$) is the beta function$

Proof:

let $\xi=\exp \left\{-\frac{\alpha}{x}-\theta\left(\frac{1}{x}\right)^{2}\right\}$ and substituting (3) and

(4) in (22), we obtain

$$
f_{r: n}(x)=\frac{\xi^{r}(1-\xi)^{n-r}\left(\alpha+\frac{2 \theta}{x}\right)\left(\frac{1}{x}\right)^{2}}{B(r, n-r+1)}
$$


$f_{r: n}(x)=n\left(\begin{array}{c}n-1 \\ r-1\end{array}\right) \sum_{p=0}^{n-r}\left(\begin{array}{c}n-r \\ p\end{array}\right)(-1)^{p} \xi^{r+p}\left(\alpha+\frac{2 \theta}{x}\right)\left(\frac{1}{x}\right)^{2}$

Using (24), the kth moment of rth order statistics of $x_{(r)}$ is given by

$\mu_{k}^{(r: n)}=n\left(\begin{array}{l}n-1 \\ r-1\end{array}\right) \sum_{p=0}^{n-r} \sum_{q=0}^{\infty}(-1)^{p+q} \mathrm{H}(k, p, q) \sum(k, p, q)$

where

$\mathrm{H}(k, p, q)=\left(\begin{array}{c}n-r \\ p\end{array}\right)\left(\frac{1}{\alpha(r+p)}\right)^{2 q-k} \frac{(\theta(r+p))^{q}}{q !}$

$\sum_{k, p, q}=\frac{1}{r+p} \Gamma(2 q-k+1)+\frac{2 \theta}{(\alpha(r+p))^{2}} \Gamma(2 q-k+2)$

\section{ESTIMATION}

Consider the random samples $\mathrm{x}_{1}, \mathrm{x}_{2}, \mathrm{x}_{\mathrm{n}}$ consisting of $\mathrm{n}$ observations from the MIR distribution $\operatorname{MIR}(x ; \alpha, \theta)$ having probability density function. The likelihood function of (3) is given by

$$
L=\prod_{i=0}^{n}\left(\alpha+\frac{2 \theta}{x}\right)\left(\frac{1}{x}\right)^{2} \exp \left\{-\frac{\alpha}{x}-\theta\left(\frac{1}{x}\right)^{2}\right\}
$$

By taking logarithm of (26), we find the log-likelihood function $\mathcal{L}=\ln L$, differentiating (27) with respect to $\alpha$ and $\theta$ then equating it to zero, we obtain the estimating equations are

$$
\begin{aligned}
& \log L=\sum_{i=0}^{n}\left(\alpha+\frac{2 \theta}{x}\right)+\sum_{i=0}^{n}\left(\frac{1}{x}\right)^{2}-\sum_{i=0}^{n}\left(\frac{\alpha}{x}\right)-\theta \sum_{i=0}^{n}\left(\frac{1}{x}\right)^{2} \\
& \frac{\partial \log L}{\partial \alpha}=\sum_{i=0}^{n}\left(\alpha+\frac{2 \theta}{x}\right)^{-1}+\sum_{i=0}^{n}\left(\frac{1}{x}\right) \\
& \frac{\partial \log L}{\partial \theta}=\sum_{i=0}^{n}\left(\alpha+\frac{2 \theta}{x}\right)^{-1}\left(\frac{2}{x}\right)-\sum_{i=0}^{n}\left(\frac{1}{x}\right)^{2}
\end{aligned}
$$$$
\frac{\partial^{2} \log L}{\partial \alpha^{2}}=-\sum_{i=0}^{n}\left(\alpha+\frac{2 \theta}{x}\right)^{-2}
$$$$
\frac{\partial^{2} \log L}{\partial \theta^{2}}=-\sum_{i=0}^{n}\left(\alpha+\frac{2 \theta}{x}\right)^{-2}\left(\frac{2}{x}\right)^{2}
$$$$
\frac{\partial^{2} \log L}{\partial \theta \partial \alpha}=-\sum_{i=0}^{n}\left(\alpha+\frac{2 \theta}{x}\right)^{-2}\left(\frac{2}{x}\right)
$$

By using (31), approximately $100(1-\alpha) \%$ confidence intervals for $\alpha$ and $\theta$ are obtained. Here $Z \alpha / 2$ is the $\alpha$ th percentile of SND . $\widehat{\alpha} \pm Z_{\alpha / 2} \sqrt{\widehat{V}_{11}}$ and $\hat{\theta} \pm Z_{\alpha / 2} \sqrt{\widehat{V}_{22}}$

\section{APPLICATION}

This section illustrate the usefulness of the MIR distribution to real data to see how the new model works in practice. The real data set corresponds to the exceedances of flood peaks (in $\mathrm{m} 3 / \mathrm{s}$ ) of the Wheaton River near Carcross in Yukon Territory, Canada. The data consist of 72 exceedances for the years 1958-1984, rounded to one decimal. The data was analyzed by Akinsete et al. [2] and is given below

$1.7,2.2,14.4,1.1,0.4,20.6,5.3,0.7,1.9,13.0,12.0,9.3,1.4$, $18.7,8.5,25.5,11.6,14.1,22.1,1.1,2.5,14.4,1.7,37.6,0.6$, $2.2,39.0,0.3,15.0,11.0,7.3,22.9,1.7,0.1,1.1,0.6,9.0,1.7$, 7.0, 20.1, 0.4, 2.8, 14.1, 9.9, 10.4, 10.7, 30.0, 3.6, 5.6, 30.8, $13.3,4.2,25.5,3.4,11.9,21.5,27.6,36.4,2.7,64.0,1.5,2.5$, $27.4,1.0,27.1,20.2,16.8,5.3,9.7,27.5,2.5,27.0$.

Table 1. Summary Statistics for MIR, IR, IE using MLE

\begin{tabular}{|c|c|c|c|}
\hline Distribution & $\begin{array}{c}\text { Coefficient } \\
\text { of Quartile } \\
\text { Deviation }\end{array}$ & $\begin{array}{c}\text { Bowley } \\
\text { Skewness }\end{array}$ & $\begin{array}{c}\text { Percentile } \\
\text { coefficient of } \\
\text { kurtosis }\end{array}$ \\
\hline MIR & 3.142819 & 0.476172 & 0.152087 \\
\hline IR & 0.365289 & 0.306863 & 0.209577 \\
\hline IE & 2.612624 & 0.476281 & 0.152078 \\
\hline
\end{tabular}

Table 2. $\mathrm{MLE}_{\mathrm{S}}$ of the Parameters for flood peaks data

\begin{tabular}{|c|c|c|c|}
\hline Distribution & $\alpha$ & $\theta$ & $-2 \ell(. ; t)$ \\
\hline MIR & $\begin{array}{c}2.28143 \\
(0.27159)\end{array}$ & $\begin{array}{c}0.105007 \\
(0.016336)\end{array}$ & -242.1675 \\
\hline IR & - & $\begin{array}{c}0.517986 \\
(0.061045)\end{array}$ & 119.8349 \\
\hline IE & $\begin{array}{c}1.89684 \\
(0.22355)\end{array}$ & - & -226.1855 \\
\hline
\end{tabular}



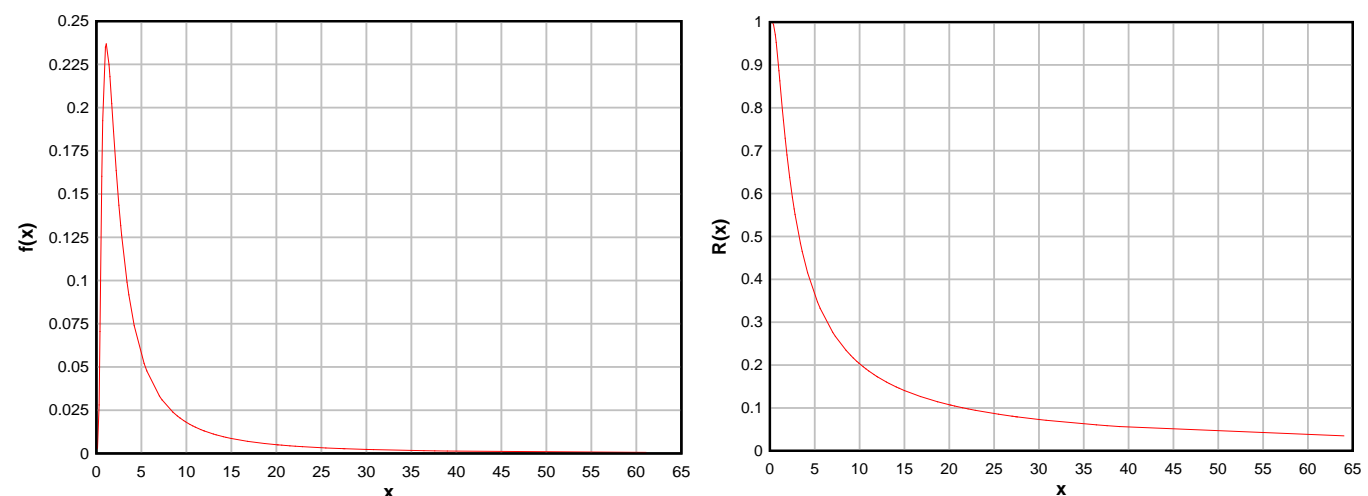

Fig 5: Fitted Modified Inverse Rayleigh for exceedances flood peaks data

Table 3. The Log-likelihood Ratio test and P-values

\begin{tabular}{|c|c|c|c|}
\hline Distribution & $\mathrm{H}_{0}$ & $\Lambda$ & P-Value \\
\hline IR & $\alpha=0$ & 362.0024 & $1.032 \mathrm{E}-80$ \\
\hline IE & $\theta=0$ & 15.982 & $6.395 \mathrm{E}-05$ \\
\hline
\end{tabular}

The summary statistics of the MIR, IR and IE distributions are discussed in Table 1 . These three distributions are fitted to the subject data using maximum likelihood estimation. The $M_{L} E_{S}$ of the parameters (with their standard errors) and their corresponding log-likelihood values are displayed in Table 2. The likelihood ratio (LR) statistics for testing the hypothesis with their corresponding p-values are discussed in Table 3. The values in table 3 indicate that the MIR distribution leads to the better fit than the IR and IE distributions. This indicates that the new parameter $\alpha$ in MIR distribution plays an important role for capturing the right skewed life time data.

\section{CONCLUSION}

This article introduces the MIR distribution, which is an extension of the IR distribution. The new parameter $\alpha$ provides more flexibility in modeling reliability data. Some of its properties are discussed illustrating the usefulness of the MIR distribution to real data using MLE. The likelihood ratio test concludes that the MIR distribution provides consistent result than the IR and IE distributions.

\section{REFERENCES}

[1] Ammar M. Sarhan and Mazen Zaindin. (2009). Modified Weibull distribution, Applied Sciences, 11, 123-136.

[2] Akinsete, A., Famoye, F. and Lee, C. (2008). The betaPareto distribution. Statistics 42, 547-563.

[3] Bonferroni C.E. (1930). Elmenti di statistica generale. Libreria Seber, Firenze
[4] Gauss M. Cordeiro, Antonio Eduardo Gomes , Cibele Queiroz da-Silva, Edwin M. M. Ortega, The beta exponentiated Weibull distribution, Journal of Statistical Computation and Simulation. 83,1, 2013, 114-138.

[5] Gharraph, M.K. (1993). Comparison of Estimators of Location Measures of an Inverse Rayleigh Distribution. The Egyptian Statistical Journal. 37, 295-309.

[6] J. F. Kenney and E. S. Keeping. Mathematics of Statistics. Princeton, NJ. (1962)

[7] Khan, M.S, King Robert, 2012 Modified Inverse Weibull Distribution, J. Stat. Appl. Pro. 1, No. 2, 115-132.

[8] Khan, M.S, Pasha, G.R and Pasha, A.H. (2008). Theoretical analysis of Inverse Weibull distribution. WSEAS Transactions on Mathematics, 7(2), 30-38.

[9] Lorenz, M. O. (1905). "Methods of measuring the concentration of wealth". The American Statistical Association, Vol. 9, No. 70) 9 (70): 209-219.

[10] Mohsin and Shahbaz (2005). Comparison of Negative Moment Estimator with Maximum Likelihood Estimator of Inverse Rayleigh Distribution, PJSOR 2005, Vol.1: $45-48$

[11] Mukarjee, S.P. and Maitim, S.S. (1996). A Percentile Estimator of the Inverse Rayleigh Parameter. IAPQR Transactions, 21, 63-65.

[12] Treyer, V. N. (1964). Doklady Acad, Nauk, Belorus, U.S.S.R.

[13] Voda, V. Gh. (1972). On the Inverse Rayleigh Random Variable, Pep. Statist. App. Res., JUSE, 19, 13-21

[14] Voda, V. G. (1975). Note on the truncated Rayleigh variate. Revista Colombiana de Matematicas, 9, 1-7.

[15] Voda, V. G. (1976). Inferential procedures on a generalized Rayleigh variate I. Applied Mathematics, 21, 395-412. 